\title{
ITAMs versus ITIMs: striking a balance during cell regulation
}

\author{
Daniel D. Billadeau ${ }^{1,2}$ and Paul J. Leibson ${ }^{2}$ \\ ${ }^{1}$ Division of Developmental Oncology Research, and \\ ${ }^{2}$ Department of Immunology, Mayo Graduate and Medical Schools, Mayo Clinic, Rochester, Minnesota, USA \\ Address correspondence to: Paul J. Leibson, Department of Immunology, \\ Mayo Clinic, 200 First Street SW, Rochester, Minnesota 55905, USA. \\ Phone: (507) 284-4563; Fax: (507) 284-1637; E-mail: leibson.paul@mayo.edu. \\ J. Clin. Invest. 109:161-168 (2002). DOI:10.1172/JCI200214843.
}

Functional immune responses evolve through an exquisitely controlled process integrating signals from activating and inhibitory receptors on the immune cell surface. These complex interactions, which regulate both the quality and magnitude of the ultimate response, depend crucially on two short, loosely conserved motifs found in the intracellular domain of various signaling proteins. These motifs, termed "ITAMs" and "ITIMs" for immunoreceptor tyrosine-based activation (or inhibititory) motifs, provide the basis for two opposed signaling modules that duel for control of cellular activation within the immune system.

Well over a decade ago it was observed that the subunits associated with the $\mathrm{B}$ cell and $\mathrm{T}$ cell antigen receptors, and the FcERI receptor shared a sequence motif now known as an ITAM $(1,2)$, a sequence postulated to link antigenic receptors to their intracellular signaling cascades (1). Since then, numerous studies have demonstrated the importance of these receptor subunits and their ITAMs in generating signals downstream of their associated receptors, providing a framework for understanding how ITAMs regulate immune cell activation. Following the identification of the ITAM, it was observed that the Fc $\gamma$ RIIB receptor could inhibit signals through the $B$ cell receptor (BCR) (3), an activity that depended on the presence of an ITIM within the Fc $\gamma$ RIIB cytoplasmic tail. Recruitment of specific phosphatases to the tyrosine-phosphorylated ITIM results in the complete inhibition or downmodulation of immune cell effector functions (reviewed in ref. 4). Over the past several years, transmembrane receptors containing ITIMs have been identified on virtually all cells in the immune system and also on some nonhematopoietic cells (reviewed in ref. 4). Interestingly, most of these ITIM-containing transmembrane receptors have homologous activating receptors that associate with ITAM-containing subunits. In this Perspective, we review the molecular interactions of ITAM- and ITIM-bearing proteins and consider their importance in regulating the outcome of immune cell stimulation by antigens.

\section{ITAMs}

The activation of many immune cell types occurs through multisubunit cell-surface receptors in which antigen-recognizing or ligand-binding subunits are noncovalently associated with one or more transmembrane adapter molecules. These transmembrane adapter molecules contain a short extracellular region, a transmembrane segment, and a cytoplasmic tail of varying length. Adapters associated with the $\mathrm{T}$ cell receptor (TCR), BCR, several activating natural killer (NK) cell receptors, some Fc receptors, and other receptors on hematopoietic cells help transduce signals to these various cell types. Common structural features of these adapter molecules are a conserved aspartic residue within the transmembrane region, which is important for association with receptor-binding subunits, and one or more copies of the ITAM, whose loose consensus sequence is $\mathrm{YXXL} / \mathrm{I}\left(\mathrm{X}_{6-8}\right) \mathrm{YXXL} / \mathrm{I}$ (where $\mathrm{X}$ denotes any amino acid) $(1,2)$. Numerous reports have highlighted the importance of the ITAM in the generation of intracellular biochemical signaling cascades following receptor engagement (reviewed in refs. $5,6)$. Depending on the maturation state of the cell or the specific cell type, these signaling cascades can culminate in such diverse biological readouts as developmental maturation, cell death, cell survival, or effector functions including cytokine production and cellular cytotoxicity.

The family of ITAM-containing proteins includes the TCR-associated CD $3 \gamma, \mathrm{CD} 3 \delta, \mathrm{CD} 3 \varepsilon$, and $\zeta$ chains; the BCR-associated $\operatorname{Ig} \alpha$ and $\operatorname{Ig} \beta$ chains; the FceRI $\beta$ chain; the FcERI-, the Fc $\gamma \mathrm{RI}$-, and the Fc $\gamma \mathrm{RIII}$-associated $\gamma$ chain; DAP12; and several virally encoded transmembrane molecules. In general, the most proximal and requisite event upon receptor engagement is the activation of Src family protein tyrosine kinases (PTKs; see ref. 7 for review). Activation of Src family PTKs leads to the phosphorylation of both tyrosines within the ITAM, which, in turn, leads to the recruitment and activation of the tandem $\mathrm{SH} 2$ domain-containing Syk and ZAP-70 tyrosine kinases. The activation of Syk and/or ZAP-70 following binding to the dually phosphorylated ITAM leads to the recruitment and phos- 
phorylation of multiple signaling molecules involved in linking the engaged receptor to its downstream signaling pathways and subsequent effector functions (reviewed in refs. 5, 6, 8).

\section{ITAM multiplicity: signal amplification or specific effector activation?}

The observation that the TCR, BCR, and some Fc receptors are associated with multiple ITAM-signaling subunits suggests that these subunits support the efficient signal amplification upon receptor engagement. Alternatively, the pairing of distinct ITAM-containing subunits could allow activated receptors to link in a modular manner to specific downstream signaling pathways by binding to distinct $\mathrm{SH} 2$ domain-containing effector molecules.

Probably the most thoroughly studied of the ITAM containing-receptors is the TCR complex, consisting of a pair of antigen-recognizing chains $(\alpha \beta$ or $\gamma \delta)$, the $\mathrm{CD} 3$ chains $(\varepsilon \gamma$ and $\varepsilon \delta)$, and a homodimeric pair of $\zeta$ chains (Figure 1). In total, the TCR complex contains ten ITAMs, one from each of the CD3 chains and six from the $\zeta$ dimer. By contrast, other ITAM-containing receptors have two to four ITAMs each. The role of the $\mathrm{CD} 3$ and $\zeta$ chains in regulating $\mathrm{T}$ cell development has been firmly established, and we recommend the recent review by Love and Shores (9) on this subject.

To explore the role of these ITAMs in TCR function, several groups have transduced $\zeta$ chains lacking one or more of these sequences into TCR transgenic, $\zeta$ chain-deficient mice. In all cases, it appears that peripheral $T$ cells from the $\zeta$ chain-reconstituted animals can still be activated through the TCR (reviewed in refs. 9, 10), suggesting that ITAMs present in the $\mathrm{CD} 3$ chains are sufficient for this process. Indeed, different CD3 chain ITAMs have been found to interact with distinct substrates in vitro (reviewed in ref. 6), implying that there is some potential for activating distinct signaling cascades through these various motifs. Thus, as Sommers et al. have noted, ITAMmutant $\mathrm{CD} 3 \varepsilon$ subunits placed into $\mathrm{CD} 3 \varepsilon$-deficient mice do not support $\mathrm{T}$ cell survival as the wild-type subunit does, at least in the transgenic TCR mouse line studied (11). These authors also found that $\mathrm{T}$ cell maturation appeared normal, suggesting that, as was seen in $\zeta$ chain ITAM-deficient animals, the loss of a functional CD3e ITAM leads to a quantitative but not a qualitative effect on TCR signaling (11). A recent study has shown that in $\mathrm{T}$ cells from $\mathrm{CD} 3 \delta$-deficient mice, which fail to undergo normal positive selection, TCR-induced $\zeta$ chain phosphorylation within lipid raft fractions and extracellular signal-regulated kinase (ERK) activation are defective. Interestingly, both events can be reconstituted following introduction of a CD $3 \delta$ subunit lacking not just its ITAM but its entire cytoplasmic domain (12). Consistent with this finding, mutation of a TCR $\alpha$ domain required for association with CD $3 \delta$ blocks positive selection (7). Therefore, in addition to their contribution to TCR signaling, the CD3 subunits may also act through non-ITAM domains to detect or influence TCR conformational changes occurring during ligand binding.
Although the various CD3 subunits appear to have similar activities, other ITAM-containing subunits may engage different signaling pathways. For instance, the FcERI $\beta$ and FcERI $\gamma$, which are both required for efficient activation of mast cells through FceRI (reviewed in ref. 13), act in concert to stimulate the PTK activity of Syk. FceRI $\beta$ associates with Lyn and, upon activation, phosphorylates FcERI $\gamma$, which then recruits and activates Syk. The biological activities of the Ig-associated Ig $\alpha$ and $\operatorname{Ig} \beta$ subunits, which also bear ITAMs in their cytoplasmic domains, remain somewhat controversial, apparently because they bind distinct sets of kinases (14). Chimeric receptors containing the $\operatorname{Ig} \alpha$ and $\operatorname{Ig} \beta$ signaling domains have been used to identify the sequences that mediate receptor coupling with different intracellular signaling cascades (reviewed in refs. 6, 15). Surprisingly, early transgenic experiments in mice suggest that the two subunits are functionally redundant $(16,17)$. In an attempt to resolve this discrepancy, Reichlin et al. (18) have tested B cell maturation in mice lacking the $\operatorname{Ig} \beta$ cytoplasmic tail $(\operatorname{Ig} \beta \Delta C)$ and have found that these cells indeed differ from their Ig $\alpha$ cytoplasmic tail $(\operatorname{Ig} \alpha \Delta C)$ deletion counterparts. In the former animals, B cell development occurs normally up to the immature B cell stage, and the B cells show normal calcium signaling upon BCR cross-linking, but they die by apoptosis prior to leaving the bone marrow (18). In homozygous Ig $\alpha \Delta \mathrm{C}$ mice, by contrast, pre-B cell development and allelic exclusion occur normally, but there is a progressive and quite substantial loss of B cells throughout all stages of development after the pre- $\mathrm{B}$ cell stage (19). Taken together, these data imply that the presence of at least one functional ITAM-containing signaling subunit within the BCR is enough to drive early $B$ cell development but is inadequate for the maintenance of B cell homeostasis. Significantly, $\operatorname{Ig} \beta^{\Delta C / \Delta C}$ mice carrying homozygous $\mathrm{Y} \rightarrow \mathrm{F}$ ITAM-inactivating mutations at the Ig $\alpha$ loci $\left(\operatorname{Ig} \alpha^{F / F F}\right)$ demonstrate a complete block in B cell development at the pro-B cell stage (20). Thus, although there appears to be significant functional redundancy between the Ig $\alpha$ and $\operatorname{Ig} \beta$ ITAM, there is a clear requirement for at least one functional ITAM in the pre-BCR at the pro- to pre-B cell transition.

The data above are consistent with the observed differences in signaling potential between chimeric Ig $\alpha$ and $\operatorname{Ig} \beta$ receptors transfected into $T$ and $B$ cell lines. They fit a model in which phosphorylation of the $\operatorname{Ig} \alpha$ and $\operatorname{Ig} \beta$ subunits occur in a set sequence, with the proximal tyrosine in the Ig $\alpha$ ITAM being the first residue to be modified (15). In addition, the presence of additional non-ITAM tyrosines within Ig $\alpha(1)$ and the serine and threonine phosphorylation of $\operatorname{Ig} \beta(15)$ suggest that these two subunits play other roles in B cell development and activation. This in part appears to be true, in that the $I g \alpha^{F F / F F}$ mouse, which still retains non-ITAM tyrosines within the Ig $\alpha$ cytoplasmic tail, shows a less severe block in B cell development than does the $\operatorname{Ig} \alpha^{\Delta C / \Delta C}$ mouse. $\operatorname{Ig} \alpha^{F F / F F}$ mice exhibit a nearly normal level of BCR-induced tyrosine phosphorylation of $\operatorname{Ig} \beta$ and Syk, but impaired Lyn tyrosine phosphorylation. Ig $\alpha$ is also tyrosine-phosphorylated in this strain, indicating that other tyrosines within the cytoplasmic tail 
are targets of BCR-activated kinases and may play an important role during B cell development and activation. Indeed, the B cell adapter protein BLNK (SLP-65) has been found to associate through its $\mathrm{SH} 2$ domain with a non-ITAM phosphorylated tyrosine within the Ig $\alpha$ cytoplasmic tail (21). Whether this interaction represents an important event in BCR-stimulated maturation or activation of $B$ cells awaits the generation of the appropriate mutant mice. In addition, mice harboring $\mathrm{Y} \rightarrow \mathrm{F}$ mutations within the ITAMs of $\operatorname{Ig} \beta$ will be important in determining the contribution of $\operatorname{Ig} \beta$ cytoplasmic tail-associated serine/threonine phosphorylation to B cell development and activation.

\section{Receptor affinity and ITAMs}

In general, $\mathrm{T}$ cells undergo positive and negative selection during development in the thymus, based on the affinity of the TCR for peptides presented in the context of endogenous MHC. Recent data suggest that $\mathrm{T}$ cell activation correlates with the "dwell-time" of the TCR-peptide-MHC complex, with shorter and longer interactions adversely affecting $\mathrm{T}$ cell activation (22). This type of activation is consistent with the kinetic proofreading model, which suggests that a minimal half-life of the TCR-peptide/MHC complex is required for complete $\mathrm{T}$ cell signaling.

The contribution of the TCR-associated ITAM-containing subunits to activation during this critical period is a matter of intense investigation. Several studies have shown that the selection of thymocytes is defective in TCR transgenic, $\zeta$-deficient animals (reviewed in ref. 9). Indeed, a TCR high-affinity ligand that promotes negative selection in mice containing three or one functional $\zeta$ ITAM nevertheless drives positive selection of $T$ cells in mice lacking $\zeta$ ITAMs (9, 10). The finding that peripheral $\mathrm{T}$ cells from $\zeta$-deficient animals are autoreactive suggests that they were selected during their development in the thymus for a strong TCR/MHC self-peptide interaction (reviewed in ref. 9). Interestingly, $\zeta$-deficient $\mathrm{T}$ cells have lower levels of surface TCR, which may account for their requirement for high-affinity autoreactive TCR-peptide/MHC interactions.

Taken together, the above data suggest that in the absence of functional $\zeta$ chain, ITAMs present in the remaining $\mathrm{CD} 3$ chains are capable of driving positive thymocyte selection. However, it appears that positive selection in the absence of the $\zeta$ chain can only occur if the affinity of the TCR ligand interaction is strong enough to reach the threshold of activation required for the selection of wild-type cells. Therefore, the presence of $\zeta$, although not required for qualitative $T$ cell activation per se, may play a critical role in gauging the TCR/ligand interaction during thymocyte development and in transducing a signal of the appropriate strength. The data also suggest that the multiplicity of the ITAMs in the TCR is required for the positive selection of a nonautoreactive MHC-restricted TCR repertoire. Determining whether or not the six ITAMs in the $\zeta$ chains mediate positive and negative selection and $\mathrm{T}$ cell activation in the absence of functional CD $3 \varepsilon \delta \gamma$ ITAMs awaits the generation of the appropriate mice.
Like the TCR, the BCR has the capacity for differential signaling based on its affinity for a given antigen. Kouskoff et al. (23) recently showed that a high- but not a low-affinity BCR/ligand interaction can promote early B cell activation signals, such as Syk and Ig $\alpha$ tyrosine phosphorylation, IL-2 production, the production of germline transcripts from the $\gamma 1$ switch region, and $\mathrm{Ca}^{2+}$ mobilization (23). Low-affinity interactions are not inert, however, but rather activate distinct signals, which promote IL-6 secretion, increase MHC class II levels, and activate Lyn to a modest extent (23). Because phosphorylation of the ITAM tyrosines within the Ig $\alpha$ and $\operatorname{Ig} \beta$ by Lyn occurs in a sequential fashion, starting with the amino-terminal tyrosine in $\operatorname{Ig} \alpha(15)$, it is intriguing to speculate that Lyn activation following low-affinity BCR interaction induces a low level of monophosphorylated ITAMs within Ig $\alpha$, perhaps recruiting other $\mathrm{SH} 2$ domain-containing proteins that could modulate the response through the receptor. The possible role of SH2-containing phosphatases in such an interaction is discussed below.

Interactions between ITAM-containing receptors can also antagonize each other's effects, as has been seen in T cells artificially expressing two distinct TCRs. Dittle et al. (24) found that the interaction of one TCR with an antagonist can inhibit signaling by the other TCR when interacting with its agonist. These authors found that the SH2 domain-containing phosphatase SHP-1 is recruited to both an antagonist-ligated and an agonist-unligated receptor (24). The mechanism of SHP-1's interaction with the TCRs, as well as its ability to antagonize the agonist-ligated receptor, will need to be further investigated. The possibility that monophosphorylated ITAMs exist in the TCR complex is controversial, but such structures could help explain this form of cross-talk between TCR molecules, since they could recruit a distinctive set of $\mathrm{SH} 2$-containing proteins (including, perhaps, SHP-1), different from those that associate with a fully activated receptor complex. Indeed, one report shows that chimeric $\zeta$ chain receptors that carry only a single phosphorylatable site in their ITAMs are effective inhibitors of signaling through the TCR (25). Two other studies that employed similarly mutated $\zeta$ chains, transfected into a $\zeta$-deficient $T$ cell line or expressed as a transgene in a $\zeta$-null background, found no such dominant inhibitory effect $(26,27)$. The reason for this discrepancy is at the moment not apparent, but it may be the result of ectopic expression of mutant $\mathrm{CD}-8 \zeta$ chimeric molecules in T cells containing functional TCR complexes.

In the FceRI system, interaction between the receptor and a low-affinity ligand leads to sequestration of Lyn and thereby prevents signaling through a highaffinity receptor interaction in the same cell (28). Conversely, in their analysis of TCR inhibition, Dittle et al. (24) did not observe sequestration of Lck to the antagonist-ligated receptor but instead observed that Lck was recruited to the agonist-ligated receptor and that the recruited Lck was inactive. It is possible that Lck is inactivated by SHP-1 which is recruited to the same TCR complex following antagonist stimulation (24). Whether SHP-1 plays a similar role in regulating 
responses to low-affinity or antagonist-mediated responses downstream of other ITAM-containing receptors remains to be investigated. Clearly, the activation of SHP-1 by ITIM-containing receptors has profound effects on ITAM-mediated signals (see below). Therefore, as in $\mathrm{T}$ cell activation, optimal activation through the BCR and FceRI fits with the kinetic proofreading model in that the duration of the interaction of the engaged receptor with its ligand will ultimately determine whether or not the cell becomes activated.

\section{ל-ITAM phosphorylation}

In naive and resting $T$ cells, the $\zeta$ chain exists in a phosphorylated form, termed the "p21" state because of its apparent mobility on SDS-PAGE, in which it associates with up to four molecules of the inactive ZAP-70 protein. Upon TCR engagement with agonist there is an increase in the $\mathrm{p} 21$ form of $\zeta$, and the protein undergoes further phosphorylation to a "p23" form. The appearance of $\mathrm{p} 23$ correlates with the tyrosine phosphorylation and activation of ZAP-70. Interestingly, partial agonists and antagonists, which favor the formation of $\mathrm{p} 21$ rather than $\mathrm{p} 23$, leave the associated ZAP-70 in an unphosphorylated state (reviewed in ref. 29). There is, however, some confusion concerning the order of phosphorylation of the tyrosines within the $\zeta$ ITAMs upon TCR-induced activation, and the identity of the phosphorylated tyrosines within the p21 form of $\zeta$. Recent studies using several different elegant approaches suggest that the p 21 form of $\zeta$ is dually phosphorylated at the two distal ITAMs (tyrosines 3,4 and 5,6; see Figure 1) and that, upon TCR engagement, the membrane-proximal ITAM (tyrosines 1,2) is phosphorylated to produce the $\mathrm{p} 23$ form (26). Phosphorylation of sites 1,2 within the amino-terminal $\zeta$ ITAM apparently requires intact tyrosines within the third $\zeta$ ITAM $(26,27)$. However, earlier work, using antibodies that were both phosphospecific and ITAM-specific, suggested a different pathway, in which the p 21 version occurs in an ordered fashion, beginning with phosphorylation at tyrosines 3 (middle ITAM) and 6 (distal ITAM) (30). One point that is not in dispute is that the $\mathrm{p} 23$ version of $\zeta$ is fully phosphorylated at all ITAM tyrosines $(26,30)$. The significance of $\zeta$ chain phosphorylation and its role in thymocyte selection and $T$ cell activation will most likely continue to be an area of intense investigation.

The activation of Src kinases such as Lck and Fyn following an appropriate signal through the TCR is likely to serve one or more of three possible purposes. First, these kinases could produce the $\mathrm{p} 23$ form by phosphorylating the remaining ITAM sites on $\zeta$. Second, they could act on the remaining CD3-ITAMs or on other $\zeta$ chains to yield more of the p 21 and p23 forms (see Figure 1). Finally, they could phosphorylate the associated ZAP-70, activating it to produce the appropriate TCR-mediated response. Upon binding of antagonists or partial agonists, conversely, the partial activation of Src family kinases - a result of

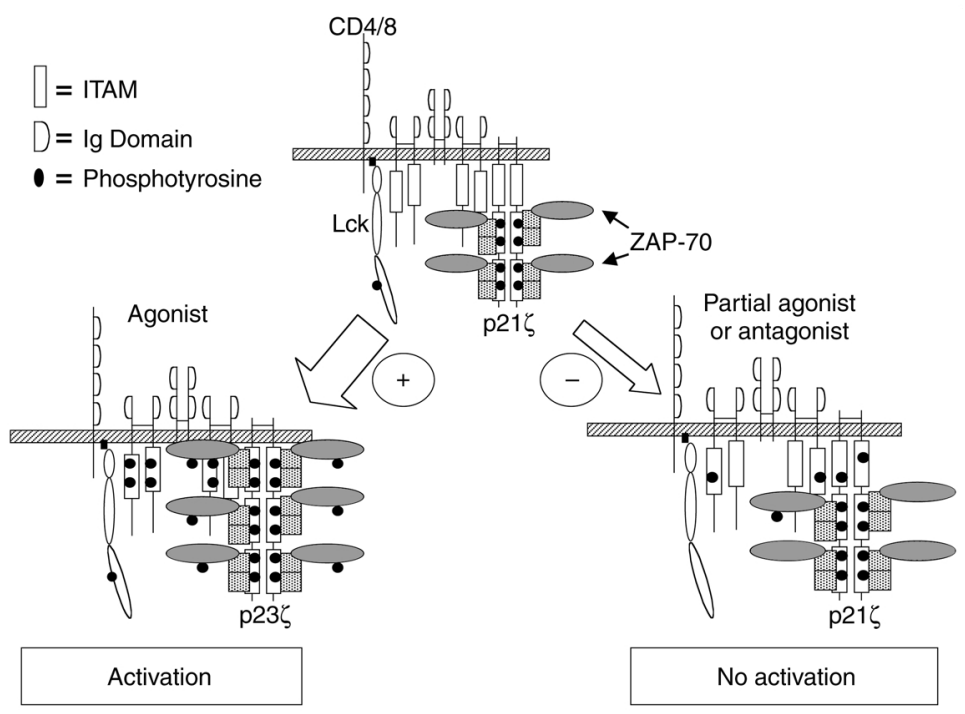

Figure 1

A model depicting TCR-mediated $\zeta$ chain phosphorylation. The figure depicts the TCR ( $\alpha$ and $\beta$ ) along with the associated CD3 chains and the 221 form of the $\zeta$ homodimer. In the resting state, some TCRs are associated with $\mathrm{p} 21 \zeta$, which bears phosphorylated tyrosines in ITAM B $(3,4)$ and $C(5,6)$ that can potentially be bound by up to four inactive ZAP-70 molecules (depicted in upper TCR complex). The Src family PTK Lck is shown myristoylated and associated with CD4 or CD8. The Src family PTK Fyn is not shown but is known to associate with the CD3E subunit. Upon agonist binding there is receptor clustering (not shown), and CD4/8-associated Lck (and CD3E-associated Fyn, not shown) can then phosphorylate the four tyrosines within the membrane-proximal ITAM (ITAM-A, tyrosines 1 and 2 ) to produce $\mathrm{p} 23 \zeta$. In addition, active Lck phosphorylates and activates bound ZAP-70, and tyrosines within the ITAMs of other CD3 subunits, resulting in the recruitment and activation of more ZAP-70 molecules (depicted in lower left). In contrast, upon binding to a partial agonist or an antagonist there is potentially less activation of Lck, which results in neither the full phosphorylation of the $\mathrm{p} 21 \zeta$ into the $\mathrm{p} 23 \zeta$ form (bottom right) nor the activation and recruitment of ZAP-70 (bottom right). Because of the blunted response through the TCR, signals that would normally result in T cell activation do not occur. Whether or not monophosphorylated ITAMs are formed and whether they serve to recruit negative regulators following partial agonist- or antagonist-TCR interaction will require further experimentation. 
the limited time with which the TCR and MHC/peptide complexes interact - allows the p21 to accumulate but does not support these later activating events.

\section{Viral ITAMs}

There is ample evidence that ITAMs are a critical component of receptor complexes involved in linking the ligand-binding portion of the receptor to the activation of intracellular signaling cascades, including those that contribute to cellular activation and cell survival. It is therefore of significant interest that a number of viruses have co-opted this signaling motif into their proteins. ITAM-containing viral proteins include bovine leukemia viruses gp30a and gp30b, Epstein-Barr virus (EBV) LMP2A, simian immunodeficiency virus $\mathrm{PBj} 14$ nef, and Kaposi sarcoma-associated herpes virus (KSHV) K1 protein. The presence of ITAMs within these proteins may play significant roles in virus production, oncogenic transformation, and disease pathogenesis. Indeed, the KSHV K1 protein has been found to oligomerize and to cause transformation when transfected into rodent fibroblasts, and a recombinant HSV containing the $K 1$ gene is sufficient to immortalize primary $\mathrm{T}$ lymphocytes and induce lymphomas. Interestingly, chimeric receptors containing an unrelated extracellular domain coupled to the intracellular region of $\mathrm{K} 1$ induce tyrosine phosphorylation of a number of cell signaling molecules and activate calcium mobilization following receptor cross-linking. These events require an intact ITAM within the K1 sequence (31). Likewise, in wild-type and RAG-deficient mice, expression of a transgene encoding the EBV LMP2A protein, but not a corresponding ITAM mutant, leads to aberrant development of B cells in which the cells fail to express any surface BCR. Hence, the LMP2A protein can provide both developmental and survival signals to B cells (32). Lyn and Syk are associated with the constitutively phosphorylated LMP2A via a single phosphorylated tyrosine and the tyrosines within the ITAM, respectively. It is of interest that, in B cells expressing LMP2A, BCR-mediated signal transduction is severely compromised. A recent study has shed some light on this phenomenon. Dykstra et al. (33) found that LMP2A resides in lipid rafts and blocks BCR translocation into these membrane domains, thereby inhibiting both BCR signaling and the internalization of the antigen-bound BCR (33). Clearly, understanding the mechanism by which these and other virally encoded ITAM-containing proteins transmit signals will be important in elucidating their pathogenic roles.

\section{ITIMs}

There is an increasing recognition that the attenuation or termination of many immune responses is not simply due to the loss of activating signals. The emerging paradigm focuses on how the balance between positive and negative signals determines functional outcomes $(4,34)$. Specifically, the co-ligation of ITAM-containing receptors with ITIM-containing coreceptors can result in a reduced or absent cellular response. The activating and inhibitory receptors may recognize either common or distinct ligands. ITIMs share a consensus amino acid

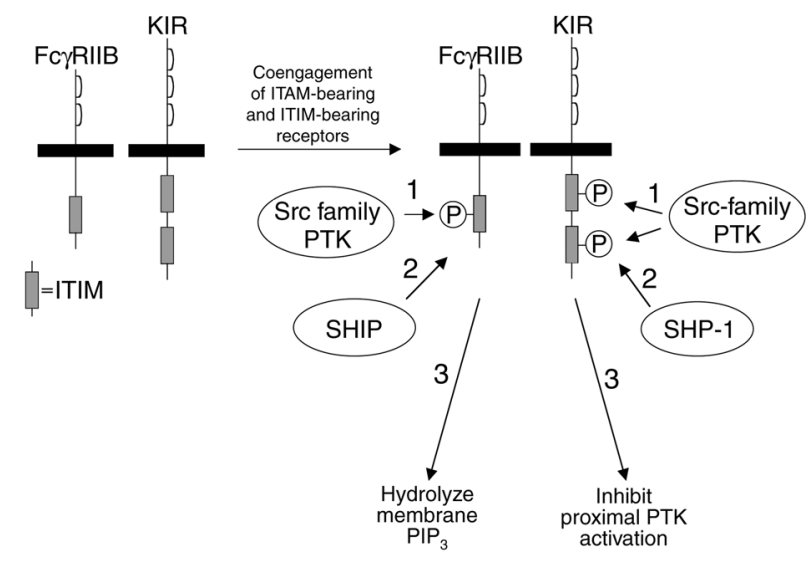

Figure 2

A model of ITIM-related cellular regulation. Co-ligation of ITAM-containing activating receptors with ITIM-bearing inhibitory receptors facilitates the Src family PTK-dependent tyrosine phosphorylation of the ITIMs. For FcyRIIB, this creates high-affinity epitopes for $\mathrm{SH} 2$-containing SHIP and the subsequent hydrolysis of the membrane inositol phosphate $\mathrm{PIP}_{3}$. For killer cell Ig-like receptors (KIRs), the tyrosine-phosphorylated ITIMs recruit SH2-containing SHP-1 (and SHP-2), leading to a block in early PTK activation.

sequence in their cytoplasmic tail, namely $(\mathrm{I} / \mathrm{V} / \mathrm{L} / \mathrm{S})-\mathrm{X}$ $\mathrm{Y}-\mathrm{X}-\mathrm{X}-(\mathrm{L} / \mathrm{V})$, where $\mathrm{X}$ denotes any amino acid $(4,35,36)$. Upon coclustering with an activating, ITAM-containing receptor, Src family PTKs phosphorylate the tyrosine residue in the ITIM sequence (Figure 2$)(4,36,37)$. The phosphotyrosine can then, broadly speaking, recruit either of two SH2 domain-containing negative regulators: the inositol phosphatase SHIP (Src homology 2-containing inositol polyphosphate 5-phosphatase) (4) or the tyrosine phosphatase SHP-1 (Src homology 2-containing protein tyrosine phosphatase-1) (36). A leucine in the $(\mathrm{Y}+2)$ position favors binding to SHIP, whereas an isoleucine in the (Y-2) position favors SHP-1 binding $(38,39)$. Some ITIMs can also bind to another tyrosine phosphatase, SHP-2, but evidence for SHP-2 playing a functional role in ITIM-mediated inhibition is less clear than for the other mediators.

The different target substrates for SHIP and SHP-1 initiate inhibitory signals through different pathways. SHIP can hydrolyze the membrane phosphoinositide $\mathrm{PIP}_{3}$, thus releasing from the membrane pleckstrin homology domain-containing (PH domain-containing) molecules such as Bruton's tyrosine kinase (Btk) and phospholipase $\mathrm{C}-\gamma 1$. Thereafter, calcium signaling is suppressed due to a decrease in the influx of calcium from extracellular stores through a capacitance-coupled channel $(4,40)$. In contrast, SHP-1 directly removes phosphate groups from various substrate proteins, thus reverting to some of the earliest events that follow upon receptor signaling and the activation of PTKs $(37,41)$.

\section{FcyRIIB-mediated inhibition}

The inhibitory IgG Fc receptor Fc $\gamma \mathrm{RIIB}$ was the first discovered and remains the best-studied example of an ITIM-containing receptor (4). Although it is broadly expressed on hematopoietic cells, its function was initially characterized in B cells, where it regulates anti- 
body production, lymphokine release, proliferation, and cell survival. Activation of SHIP recruited to the neighborhood of this receptor can abrogate calciumdependent downstream events as described above. In addition, SHIP activation prevents the recruitment of the $\mathrm{PH}$ domain-containing factor Akt and can thereby affect cell survival (42-44). Tyrosine-phosphorylated SHIP can also associate with the RasGAP-binding protein Dok because of Dok's phosphotyrosine-binding domain, leading to Ras and ERK inhibition $(45,46)$. In vivo evidence for the significance of this mechanism comes from the observation that Fc $\gamma$ RIIB-mediated inhibition of BCR-induced proliferation of mature splenic B cells does not occur in Dok ${ }^{-/}$mice (46). Homoaggregation of Fc $\gamma$ RIIB without BCR stimulation has also been proposed to generate a Btk-dependent proapoptotic signal in an ITIM-independent manner, a mechanism that may also contribute to peripheral tolerance in the humoral immune system (47). With coengagement of Fc $\gamma$ RIIB and BCR, however, SHIP recruitment to the receptor's ITIM may prevent the Btk-dependent proapoptotic signal, thus favoring B cell survival.

The prominent role of FcyRIIB as a negative regulator is underscored by the phenotype of Fc $\gamma R_{R I B}{ }^{-/-}$mice. These mice develop autoantibodies and autoimmune glomerulonephritis in a strain-dependent manner (48). In addition, $F c \gamma R I{ }^{-1-}$ mice are susceptible to collageninduced arthritis and the development of Goodpasture syndrome-like glomerular disease (49), consistent with a model in which FcyRIIB contributes to the maintenance of tolerance and protection from autoimmune disease. The negative regulatory FcyRIIB receptor might therefore be targeted therapeutically in the setting of autoimmune disease; for this reason, Samuelsson et al. (50) have tested the anti-inflammatory effect of providing gammaglobulin (the ligand for this Fc receptor) in intravenous form to mice with autoimmune thrombocytopenia (50). This so-called intravenous immunoglobulin (IVIG) therapy attenuates autoantibody-triggered inflammatory disease and prevents platelet consumption in wild-type animals, but not in those lacking the Fc $\gamma$ RIIB receptor. Therapies that enhance Fc $\gamma$ RIIB expression or activity may therefore prove useful in treating autoimmune disease.

\section{MHC-recognizing inhibitory receptors}

Whereas FcyRIIB has been studied as a prototypic model for ITIM-dependent SHIP-mediated inhibition, MHC-recognizing receptors have been extensively evaluated in studies of ITIM interactions with SHP-1. The identification of inhibitory receptors for MHC class I stemmed from earlier observations that NK cells preferentially kill tumor targets lacking MHC class I antigens (51), which suggested that some receptors for class I MHC molecules might actively suppress cellmediated killing. Three families of MHC-recognizing inhibitory receptors have now been identified on these cells: Ly49, killer cell Ig-like receptors (KIRs), and CD94/NKG2 (for review see refs. 4, 33). Ly49 molecules are type II membrane glycoproteins of the C-type lectin family that are expressed as homodimers on the cell surface of NK cells and memory T cells. Ly49 is polymorphic, and different forms recognize the various murine class I molecules. Likewise, in humans, different human KIRs recognize polymorphic epitopes on human leukocyte classes HLA-A, HLA-B, and HLA-C. The CD94/NKG2 heterodimer, which is found in both humans and mice, recognizes human HLA-E and the homologous mouse $\mathrm{Qa} 1^{\mathrm{b}}$ molecule.

Inhibitory MHC receptors of each of these classes carry ITIMs in their cytoplasmic domains that allow them to bind SHP-1 preferentially and to inhibit cellmediated killing and cytokine secretion. Each of these families also contains activating counterparts in which the cytoplasmic ITIMs are lacking and in which the transmembrane domain contains charged residues that facilitate interaction with DAP12, an ITAM-containing homodimer. Increasing data support a role for these kinds of inhibitory and activating receptors in the destruction of tumor cells lacking MHC class I, in protection against a variety of viral pathogens and in rejection of bone marrow allografts.

Although SHP-1 recruitment to the inhibitory MHCrecognizing receptors clearly blocks PTK-related proximal signaling events, it has been difficult to identify the key substrates for this phosphatase. Among the potential substrates are LAT (52) and SLP-76 (53); Chiang and Sefton (54) recently demonstrated that SHP-1 can also dephosphorylate the Src family kinase Lck at Tyr 394. Since phosphorylation of Tyr 394 activates Lck, SHP-1-mediated dephosphorylation at this site could go far toward explaining its profound suppressive effect on signaling through the TCR. Indeed, since Src family PTK activation is the earliest detectable signal generated by ITAM-containing activating receptors, the targeting of this most proximal PTK could account for the observed global and complete inhibition. This model of negative regulation is also consistent with the observations that targeting SHP-1 into lipid rafts inhibits lymphocyte activation (55) and that KIR-associated SHP-1 blocks the early PTK-dependent polarization of lipid rafts during the generation of cellmediated killing (56).

Until recently, most studies on MHC-recognizing inhibitory receptors have focused on the SHP-1-mediated blockade of cytokine secretion and cell-mediated killing. Newer data also indicate a role for these inhibitory receptors in the survival of memory $\mathrm{T}$ cells. In addition to the broad distribution of these receptors on NK cells, a subset of memory $\mathrm{CD}^{+} \mathrm{T}$ cells express inhibitory Ly49s in mice and inhibitory KIRs in humans $(4,57)$. Interestingly, memory $\mathrm{CD}^{+} \mathrm{T}$ cells accumulate in transgenic animals expressing these inhibitory receptors (58). Similarly, MHC recognition by the inhibitory receptors suppresses activation-induced cell death in vitro, highlighting the possibility that, like the SHIP-associated receptors, these ITIM-bearing coreceptors can influence cell survival. This effect might be explained by the targeting of key regulatory signals by SHP-1, or perhaps the recruitment by ITIMs of other signaling molecules, such as phosphatidylinositol 3-kinase (59). Hence, the balance between paired ITAM- and ITIM-containing receptors could critically influence cell survival. 


\section{ITIM-containing receptors on hematopoietic and nonhematopoietic cells}

The term "inhibitory receptor superfamily" has been used to describe the growing number of ITIM-bearing receptors that, upon coengagement with activating receptors, block cellular activation (4). These include negative regulators such as ILT (also known as LIR, MIR, and CD85) and PIR-B receptors, which are predominantly expressed on monocytes, macrophages, dendritic cells, and B lymphocytes (reviewed in refs. 4, 33). Additional ITIM-bearing receptors include LAIR-1 (on leukocytes), gp49B (on mast cells, macrophages, and NK cells), CD5 (on thymocytes, mature T cells, and the B-1a subpopulation of B cells), CD22 (on B cells), FDF03 (on dendritic and myeloid cells), SIRP $\alpha$ (on myeloid and nonhematopoietic cells), CD33-related receptors (on myeloid cells and lymphocytes), and MAFA (on mast cells, myeloid cells, and NK cells). In some cases, the corresponding ligands are known (e.g., IgG for FcyRIIB, MHC class I for Ly49s and KIRs, sialic acid residues for the CD33-like receptors, and CD47 for SIRP $\alpha$ ). It is clear that a broad variety of additional receptors, including some that are thought of as "activating" receptors, can bind via a modified ITIM to negative regulators (60). Such receptors are therefore distinct from those mentioned above, which inhibit activating receptors in trans.

As new ligands are identified, the range of immune responses known to be regulated by ITIM-bearing receptors increases. For example, the integrin $\alpha_{v} \beta_{3}$ was recently identified as a ligand for ITIM-bearing protein gp49B1, which is stimulated by integrin binding to inhibit mast cell activation (61). These observations suggest new potential mechanisms for the downregulation of allergic responses and, more broadly, raise the possibility that the inhibitory receptor superfamily could be relevant to the development of atopic diseases.

\section{Conclusion}

It now appears that the strength and nature of immune responses are determined by the pairing of activating and inhibitory receptors. Thus, in vivo, abnormalities in ITAM-bearing receptors can result in selective immunodeficiencies and, conversely, dysfunctional ITIM-bearing receptors can lead to potentially fatal autoimmune disorders. The range of cellular responses controlled by these paired receptors is broad and includes antibody production, cytokine production, phagocytosis, cell-mediated killing, and cellular proliferation. The evolutionary conservation of these motifs extends across the vertebrate species and, in the case of ITAMs, even beyond $(62,63)$, suggesting an even broader utilization of these signaling modules in cell regulation. Importantly, one can use our understanding of these potent regulatory mechanisms to design therapeutic strategies. Recent studies of intravenous Ig therapy highlight this exciting potential (50). Additional work will be needed to determine which forms of immunologic responses (e.g., autoimmune reactions, resistance to infection, antitumor immunity, atopic allergic reactions, etc.) will be amenable to manipulation by ITAM- or ITIM-directed therapies.

\section{Acknowledgments}

P.J. Leibson is supported by the Mayo Foundation and by NIH grant CA-47752. D.D. Billadeau is supported by the Mayo Foundation and is a Special Fellow of the Leukemia and Lymphoma Society of America. Due to space constraints, a number of important references could not be included in this article. Interested readers can find a suggested reading list at http://www.jci.org/cgi/content/full/109/2/161/dc1.

1. Reth, M. 1989. Antigen receptor tail clue. Nature. 338:383-384.

2. Cambier, J.C. 1995. New nomenclature for the Reth motif (or ARH1/TAM/ARAM/YXXL). Immunol. Today. 16:110.

3. Amigorena, S., et al. 1992. Cytoplasmic domain heterogeneity and functions of IgG Fc receptors in B lymphocytes. Science. 256:1808-1812.

4. Ravetch, J.V., and Lanier, L.L. 2000. Immune inhibitory receptors. Science. 290:84-89.

5. Weiss, A. 1993. T cell antigen receptor signal transduction: a tale of tails and cytoplasmic protein tyrosine kinases. Cell. 73:209-212.

6. Isakov, N. 1998. ITAMs: immuoregulatory scaffolds that link immunoreceptors to their intracellular signaling pathways. Receptors Channels. 5:243-253.

7. Backstrom, B.T., Muller, U., and Palmer, E. 2000. Positive selection through a motif in the $\alpha \beta \mathrm{T}$ cell receptor. Science. 281:835-838.

8. Tomlinson, M.G., Lin, J., and Weiss, A. 2000. Lymphocytes with a complex: adapter proteins in antigen receptor signaling. Immunol. Today. 21:584-591.

9. Love, P.E., and Shores, E.W. 2000. ITAM multiplicity and thymocyte selection: how low can you go? Immunity. 12:591-597.

10. Love, P.E., Lee, J., and Shores, E.W. 2000. Critical relationship between TCR signaling potential and TCR affinity during thymocyte selection. J. Immunol. 165:3080-3087.

11. Sommers, C.L., et al. 2000. Function of CD3 $\varepsilon$-mediated signals in T cell development. J. Exp. Med. 192:913-919.

12. Delgado, P., Fernandez, E., Dave, V., Kappes, D., and Alarcon, B. 2000. CD3 $\delta$ couples T-cell receptor signaling to ERK activation and thymocytes positive selection. Nature. 406:426-430.

13. Nadler, M.J., Matthews, S.A., Turner, H., and Kinet, J.-P. 2000. Signal transduction by the high affinity immunoglobulin E receptor Fc epsilon RI: coupling form to function. Adv. Immunol. 76:325-355.

14. Clark, M.R., et al. 1992. The B cell antigen receptor complex: association of Ig-alpha and Ig-beta with distinct cytoplasmic effectors. Science. 258:123-126.

15. Pao, L., Famiglietti, S.J., and Cambier, J. 1998. Asymmetrical phosphorylation and function of the immunoreceptor tyrosine-based activation motif tyrosines in B cell antigen receptor signal transduction. J. Immunol. 160:3305-3314

16. Papavasiliou, F., Jankovic, M., Suh, H., and Nussenzweig, M.C. 1995. The cytoplasmic domains of $\operatorname{Ig} \alpha$ and $\operatorname{Ig} \beta$ can independently induce the preB cell transition and allelic exclusion. J. Exp. Med. 182:1389-1394.

17. Teh, Y., and Neuberger, M.S. 1997. The immunoglobulin $\operatorname{Ig} \alpha$ and $\operatorname{Ig} \beta$ cytoplasmic domains are independently sufficient to signal B cell maturation and activation in transgenic mice. J. Exp. Med. 185:1753-1768.

18. Reichlin, A., et al. 2001. B cell development is arrested at the immature B cell stage in mice carrying a mutation in the cytoplasmic domain of immunoglobulin $\beta$. J. Exp. Med. 193:13-23.

19. Torres, R.M., Flaswinkel, H., Reth, M., and Rajewsky, K. 1996. Aberrant $B$ cell development and immune response in mice with a compromised BCR complex. Science. 272:1802-1804.

20. Kraus, M., et al. 2001. Interference with immunoglobulin (Ig) $\alpha$ immunoreceptor tyrosine-based activation motif (ITAM) phosphorylation modulates or blocks B cell development, depending on the availability of an Ig $\beta$ cytoplasmic tail. J. Exp. Med. 194:455-469.

21. Engels, N., Wollscheid, B., and Wienands, J. 2001. Association of SLP65 /BLNK with the B cell antigen receptor through a non-ITAM tyrosine

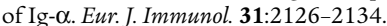

22. Kelergis, A.M., et al. 2001. Efficient T cell activation requires an optimal dwell-time of interaction between the TCR and the PMHC complex. Nat. Immunol. 2:229-234.

23. Kouskoff, V., et al. 1998. Antigens varying in affinity for the B cell-receptor induce differential B lymphocyte responses. J. Exp. Med. 188:1453-1464.

24. Dittle, B.N., Stefanova, I., Germain, R.N., and Janeway, C.A., Jr. 1999. Cross-antagonism of a $\mathrm{T}$ cell clone expressing two distinct $\mathrm{T}$ cell receptors. Immunity. 11:289-298.

25. Kersh, E.N., Kersh, G.J., and Allen, P.M. 1999. Partially phosphorylated $\mathrm{T}$ cell receptor $\zeta$ molecules can inhibit $\mathrm{T}$ cell activation. J. Exp. Med. 190:1627-1636

26. van Oers, N.S.C., et al. 2000. The 21- and 23-kD forms of TCR $\zeta$ are generated by specific ITAM phosphorylations. Nat. Immunol. 1:322-328. 
27. Ardouin, L., et al. 1999. Crippling of CD3- $\zeta$ ITAMS does not impair T cell receptor signaling. Immunity. 10:409-420.

28. Torigoe, C., Inman, J.K., and Metzger, H. 1998. An unusual mechanism for ligand antagonism. Science. 281:568-572.

29. Sloan-Lancaster, J., and Allen, P.M. 1996. Altered peptide ligand induced partial $\mathrm{T}$ cell activation: molecular mechanisms and role in $\mathrm{T}$ cell biology. Annu. Rev. Immunol. 14:1-27.

30. Kersh, E.N., Shaw, A.S., and Allen, P.M. 1998. Fidelity of T cell activation through multistep T cell receptor $\zeta$ phosphorylation. Science. 281:572-575.

31. Lee, H., et al. 1998. Identification of an immunoreceptor tyrosine-based activation motif of KI transforming protein of Kaposi's sarcoma-associated virus. Mol. Cell. Biol. 18:5219-5228.

32. Merchant, M., Caldwell, R.G., and Longnecker, R. 2000. The LMP2A ITAM is essential for providing B cells with development and survival signals in vivo. J. Virol. 74:9115-9124.

33. Dykstra, M.L., Longnecker, R., and Pierce, S.K. 2001. Epstein-Barr virus coopts lipid rafts to block the signaling and antigen transport functions of the BCR. Immunity. 14:57-67.

34. Long, E.O. 1999. Regulation of immune responses through inhibitory receptors. Annu. Rev. Immunol. 17:875-904.

35. Vivier, E., and Daeron, M. 1997. Immunoreceptor tyrosine-based inhibition motifs. Immunol. Today. 18:286-291.

36. Burshtyn, D.N., et al. 1996. Recruitment of tyrosine phosphatase HCP by the killer cell inhibitor receptor. Immunity. 4:77-85.

37. Binstadt, B.A., et al. 1996. Sequential involvement of Lck and SHP-1 with MHC-recognizing receptors on NK cells inhibits FcR-initiated tyrosine kinase activation. Immunity. 5:629-638.

38. Burshtyn, D.N., Yang, W., Yi, T., and Long, E.O. 1997. A novel phosphotyrosine motif with a critical amino acid at position -2 for the $\mathrm{SH} 2$ domain-mediated activation of the tyrosine phosphatase SHP-1. J. Biol. Chem. 272:13066-13072.

39. Bruhns, P., et al. 2000. Molecular basis of the recruitment of the SH2 domain-containing inositol 5-phosphatases SHIP1 and SHIP2 by Fc $\gamma$ RIIB. J. Biol. Chem. 275:37357-37364.

40. Scharenberg, A.M., et al. 1998. Phosphatidylinositol-3,4,5-trisphosphate (Ptdlns-3,4,5-P3)/Tec kinase-dependent calcium signaling pathway: a target for SHIP-mediated inhibitory signals. EMBO J. 17:1961-1972.

41. Brumbaugh, K.M., et al. 1996. Clonotypic differences in signaling from CD94 (kp43) on NK cells lead to divergent cellular responses. J. Immunol. 157:2804-2812.

42. Aman, M.J., Lamkin, T.D., Okada, H., Kurosaki, T., and Ravichandran, K.S. 1998. The inositol phosphatase SHIP inhibits Akt/PKB activation in B cells. J. Biol. Chem. 273:33922-33928.

43. Liu, Q., et al. 1999. SHIP is a negative regulator of growth factor receptor-mediated PKB/Akt activation and myeloid cell survival. Genes Dev. 13:786-791.

44. Helgason, C.D., et al. 2000. A dual role for Src homology 2 domain-containing inositol-5-phosphatase (SHIP) in immunity: aberrant development and enhanced function of B lymphocytes in ship -/- mice. J. Exp. Med. 191:781-794.

45. Tamir, I., et al. 2000. The RasGAP-binding protein $p 62^{\text {dok }}$ is a mediator of inhibitory Fc $\gamma$ RIIB signals in B cells. Immunity. 12:347-358.
46. Yamanashi, Y., et al. 2000. Role of the rasGAP-associated docking protein $\mathrm{p} 62^{\mathrm{dok}}$ in negative regulation of $\mathrm{B}$ cell receptor-mediated signaling. Genes Dev. 14:11-16.

47. Pearse, R.N., et al. 1999. SHIP recruitment attenuates Fc gamma RIIBinduced B cell apoptosis. Immunity. 10:753-760.

48. Bolland, S., and Ravetch, J.V. 2000. Spontaneous autoimmune disease in Fc $\gamma$ RIIB-deficient mice results from strain-specific epistasis. Immunity. 13:277-285.

49. Nakamura, A., et al. 2000. Fc $\gamma$ receptor IIB-deficient mice develop Goodpasture's syndrome upon immunization with type IV collagen: a novel murine model for autoimmune glomerular basement membrane disease. J. Exp. Med. 191:899-905.

50. Samuelsson, A., Towers, T.L., and Ravetch, J.V. 2001. Anti-inflammatory activity of IVIG mediated through the inhibitory Fc receptor. Science. 291:484-486.

51. Ljunggren, H.-G., and Karre, K. 1990. In search of the 'missing self': MHC molecules and NK cell recognition. Immunol. Today. 11:237-244.

52. Valiante, N.M., Phillips, J.H., Lanier, L.L., and Parham, P. 1996. Killer cell inhibitory receptor recognition of human leukocyte antigen (HLA) class I blocks formation of a pp36/PLC-gamma signaling complex in human natural killer (NK) cells. J. Exp. Med. 184:2243-2250

53. Binstadt, B.A., et al. 1998. SLP-76 is a direct substrate of SHP-1 recruited to killer cell inhibitory receptors. J. Biol. Chem. 273:27518-27523.

54. Chiang, G.G., and Sefton, B.M. 2001. Specific dephosphorylation of the Lck tyrosine protein kinase at Tyr 394 by the SHP-1 protein tyrosine phosphatase. J. Biol. Chem. 276:23173-23178.

55. Su, M.W.-C., Yu, C.-L., Burakoff, S.J., and Jin, Y.-J. 2001. Targeting Src homology 2 domain-containing tyrosine phosphatase (SHIP-1) into lipid rafts inhibits CD3-induced T cell activation. J. Immunol. 166:3975-3982.

56. Lou, Z., Jevremovic, D., Billadeau, D.D., and Leibson, P.J. 2000. A balance between positive and negative signals in cytotoxic lymphocytes regulates the polarization of lipid rafts during the development of cell-mediated killing. J. Exp. Med. 191:347-354.

57. Mingari, M.C., Ponte, M., Vitale, C., Bellomo, R., and Moretta, L. 2000. Expression of HLA class I-specific inhibitory receptors in human cytolytic T lymphocytes: a regulated mechanism that controls T-cell activation and function. Hum. Immunol. 61:44-50.

58. Ugolini, S., et al. 2001. Involvement of inhibitory NKRs in the survival of a subset of memory-phenotype CD8 ${ }^{+} \mathrm{T}$ cells. Nat. Immunol. 2:430-434

59. Marti, F., et al. 1998. LCK-phosphorylated human killer cell-inhibitory receptors recruit and activate phosphatidylinositol 3-kinase. Proc. Natl. Acad. Sci. USA. 95:11810-11815

60. Sinclair, N.R. 2000. Immunoreceptor tyrosine-based inhibitory motifs on activating molecules. Crit. Rev. Immunol. 20:89-102.

61. Castells, M.C., et al. 2001. Gp49B1- $\alpha_{v} \beta_{3}$ interaction inhibits antigeninduced mast cell activation. Nat. Immunol. 2:436-442.

62. Takahashi, H., Ishikawa, G., Ueki, K., Azumi, K., and Yokosawa, H. 1997. Cloning and tyrosine phosphorylation of a novel invertebrate immunocyte protein containing immunoreceptor tyrosine-based activation motifs. J. Biol. Chem. 272:32006-32010.

63. Strong, S.J., et al. 1999. A novel multigene family encodes diversified variable regions. Proc. Natl. Acad. Sci. USA. 96:15080-15085. 